\title{
Explicit expression for the photon number emission in synchrotron radiation*
}

\author{
E. B. Manoukian ${ }^{\dagger}$ and N. Jearnkulprasert \\ School of Physics, Suranaree University of Technology \\ Nakhon Ratchasima, 30000, Thailand
}

\begin{abstract}
An explicit and remarkably simple one-dimensional integral expression is derived for the mean number $\langle N\rangle$ of photons emitted per revolution in synchrotron radiation. The familiar high-energy expression $5 \pi \alpha / \sqrt{3\left(1-\beta^{2}\right)}$, printed repeatedly in the literature, is found to be inaccurate and only truly asymptotic with relative errors of $160 \%$, $82 \%$ for $\beta=0.8,0.9$, respectively. A new improved high-energy expression for $\langle N\rangle$ is given.
\end{abstract}

The fascinating story of synchrotron radiation emphasizing both the early theoretical and experimental developments is well documented in the literature (e.g., [1]). The monumental pioneering theoretical contribution of Schwinger [2, 3] and its direct experimental impact has been particularly noted [1]. Although the main features of synchrotron radiation have been well known for a long time, there is certainly room for further developments and improvements. In this Letter, we use an earlier integral for the power of radiation obtained by Schwinger [2, 3, 5] over fifty years ago to derive an explicit expression for the mean number $\langle N\rangle$ of photons emitted per revolution with no approximation made. The derived result for $\langle N\rangle$ is a remarkably simple one-dimensional integral. We infer that the familiar high-energy expression $5 \pi \alpha / \sqrt{3\left(1-\beta^{2}\right)}$ [6, 7] for $\langle N\rangle$, and repeatedly printed in the literature, is rather inaccurate and is to be considered only as truly asymptotic in the sense that even for speeds $\beta=0.9,0.8$ deviations from this expression are rather significant with large relative errors of $82 \%, 160 \%$ (!), respectively. In particular, our explicit result for $\langle N\rangle$ is used to obtain a much-improved asymptotic high-energy expression for radiating particles.

Our starting expression for $\langle N\rangle$ is obtained directly from Schwingers formulae (2], Eqs. III 6, 7; 4], Eq. (C.11)) for the power.

$$
\langle N\rangle=\frac{\alpha}{\beta} \int_{0}^{\infty} \mathrm{d} z \int_{-\infty}^{\infty} \mathrm{d} x \mathrm{e}^{-\mathrm{i} z x}\left(\beta^{2} \cos x-1\right) \frac{\sin \left(2 \beta z \sin \frac{x}{2}\right)}{\sin \frac{x}{2}} .
$$

*Published in Physics Letters A268 (2000) 35-36.

${ }^{\dagger}$ E-mail: edouard@ccs.sut.ac.th 
Since the integrand factor in (11) multiplying $\exp (-\mathrm{i} z x)$ is an even function of $x$, only the real part of the integral is non-vanishing. It is easily verified that $\langle N\rangle=0$ for $\beta=0$, as it should be, when integrating over $x$ and $z$ in (II) and using in the process that $\int_{0}^{\infty} \mathrm{d} z z \int_{-\infty}^{\infty} \mathrm{d} x \mathrm{e}^{-\mathrm{i} z x}$. Accordingly we may rewrite (11) as

$$
\begin{aligned}
\langle N\rangle=\alpha & \int_{0}^{\infty} \mathrm{d} z \int_{-\infty}^{\infty} \mathrm{d} x \mathrm{e}^{-\mathrm{i} z x} \int_{0}^{\beta} \mathrm{d} \rho \\
& \times\left(\cos x \frac{\sin \left(2 \beta z \sin \frac{x}{2}\right)}{\sin \frac{x}{2}}-\frac{2 z}{\beta}\left[\cos \left(2 z \rho \sin \frac{x}{2}\right)-1\right]\right)
\end{aligned}
$$

conveniently written by taking into account the explicit vanishing property of $\langle N\rangle=0$ for $\beta=0$. To evaluate $\langle N\rangle=0$, we first integrate over $z$, then over $\rho$ to obtain

$$
\langle N\rangle=\alpha \int_{-\infty}^{\infty} \mathrm{d} x\left\{\frac{2\left(1-\beta^{2} \cos x\right)}{\left(x^{2}-4 \beta^{2} \sin ^{2} \frac{x}{2}\right)}-\frac{2}{x^{2}}\right\} .
$$

Upon a change of variable $x / 2 \rightarrow x$, we finally obtain the remarkably simple expression

$$
\begin{aligned}
\langle N\rangle & =2 \alpha \beta^{2} \int_{0}^{\infty} \frac{\mathrm{d} x}{x^{2}}\left[\frac{\left(\frac{\sin x}{x}\right)^{2}-\cos (2 x)}{\left(1-\beta^{2}\left(\frac{\sin x}{x}\right)^{2}\right)}\right] \\
& \equiv \alpha f(\beta) .
\end{aligned}
$$

There is no question of the existence of the latter integral for all $0 \leq \beta<1$. [For completeness we provide values for $f(\beta)$ : 0.1731, 0.7694, 2.1351, 5.7951, 11.4003, 54.7651 corresponding, respectively, to $\beta=0.2,0.4,0.6,0.8,0.9,0.99$.]

Eq. (41) leads to the following expression :

$$
\begin{aligned}
f(\beta) & =f_{0}(\beta)+a_{0}+\varepsilon(\beta) \\
f_{0}(\beta) & =10 \beta \int_{0}^{\infty} \mathrm{d} x\left[3\left(1-\beta^{2}\right)+\beta^{2} x^{2}\right]^{-1} \\
& =\frac{5 \pi}{\sqrt{3\left(1-\beta^{2}\right)}} . \\
a_{0} & =2 \int_{0}^{\infty} \frac{\mathrm{d} x}{x^{2}} \frac{\left[6\left(\frac{\sin x}{x}\right)^{2}-\cos (2 x)-5\right]}{\left[1-\left(\frac{\sin x}{x}\right)^{2}\right]} \\
& =-9.5580 .
\end{aligned}
$$


For $\beta \rightarrow 1$

$$
\varepsilon(\beta)=\mathcal{O}\left(\sqrt{1-\beta^{2}}\right) .
$$

That is, at high energies we may write

$$
\langle N\rangle \cong \frac{5 \pi \alpha}{\sqrt{3\left(1-\beta^{2}\right)}}+a_{0} \alpha .
$$

The asymptotic constant $a_{0}$ is overwhelmingly large in magnitude. It is the important contribution that survives in the limit $\beta \rightarrow 1$ beyond the $1 / \sqrt{1-\beta^{2}}$ term. Eq. (9) provides a significantly much improved high-energy expression for $\langle N\rangle$. The relative errors in (9) are quite satisfactory with $4.11 \%, 1.34 \%, 0.063 \%$ for $\beta=0.8,0.9,0.99$. They are to be compared with the relative errors of $160 \%, 82 \%, 17 \%$, respectively, for the truly asymptotic earlier formula.

\section{References}

[1] H. C. Pollock, Am. J. Phys. 51 (3), 278 (1983).

[2] J. Schwinger, Phys. Rev. 75, 1912 (1949).

[3] J. Schwinger, Phys. Rev. 70, 798 (1946).

[4] J. Schwinger and W.-Y. Tsai, Ann. Phys. (NY) 110, 63 (1978).

[5] J. Schwinger, W.-Y. Tsai and T. Erber, Ann. Phys. (NY) 96, 303 (1976).

[6] Review of Particle Physics, Eur. Phys. J. 3, 79 (1998).

[7] R. M. Barnett et al., Review of Particle Physics, Phys. Rev. D54 (1996) 75. 